INRA Prod. Anim.,

2014,27 (2), 147-160

\title{
Le processus d'innovation en ferme. Illustrations en élevage
}

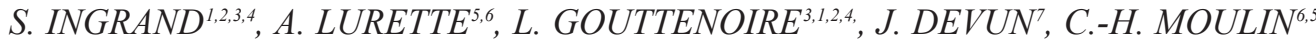 \\ 1 INRA, UMR1273 Métafort, F-63122 Saint-Genès-Champanelle, France \\ 2 AgroParisTech, UMR1273 Métafort, F-63170 Aubière, France \\ 3 Clermont Université, VetAgro Sup, UMR1273 Métafort, F-63370 Lempdes, France \\ ${ }^{4}$ Irstea, UMR1273 Métafort, F-63170 Aubière, France \\ 5 INRA, UMR868 Selmet, 2 Place Viala, F-34060 Montpellier, France \\ 6 SupAgro, UMR868 Selmet, 2 Place Viala, F-34060 Montpellier, France \\ 7 Institut de l'Elevage, UMT Safe, 9 Allée Pierre de Ferma, F-63170 Aubière, France
}

Courriel : stephane.ingrand@clermont.inra.fr

Les innovations peuvent avoir différentes origines, dont les éleveurs eux-mêmes, qui ont des connaissances, empiriques pour certaines, qu'il convient d'intégrer dans les travaux de recherche. Le processus d'innovation relève d'un processus complexe impliquant des acteurs mus par des logiques variées, avec des modifications induites dans les systèmes plus ou moins radicales. Nous donnons ici quelques exemples choisis en élevage.

L'innovation, dans le sens courant, est très souvent assimilée à l'objet nouveau introduit dans une organisation. De nombreuses publications scientifiques se centrent ainsi sur ces nouveaux objets et en produisent des analyses détaillées. L'innovation est alors le résultat du travail de conception de cet objet. Cependant, l'innovation ne correspond pas à un instant précis, celui de sa diffusion sur le marché, ou bien celui de la fin de son développement technique, mais doit être envisagée en tant que processus qui va de l'invention à sa diffusion parmi les utilisateurs ne l'ayant pas conçue euxmêmes. Gonard et Louzel (1997) y ajoutent le caractère collectif du processus. Selon eux, l'innovation ne peut être portée par un individu isolé, mais doit être vue comme le résultat d'un travail collectif. Ce n'est plus alors l'objet de l'innovation qui prime, mais la façon dont elle diffuse dans le tissu social (Cros 1997). Si les innovations peuvent être initiées à l'extérieur du réseau des utilisateurs pressentis, certaines sont produites à l'intérieur de ce réseau et peuvent émerger du groupe social lui-même. Ce sont les disciplines de l'économie, des sciences de gestion et des sciences humaines, avec notamment la sociologie, qui considèrent l'innovation comme un processus et apportent le plus de précisions sur le concept (Flichy 1995). Ces travaux montrent que l'innovation est une notion complexe et qu'elle peut être définie de différentes manières (Beguin et al 2008).

Ce cadre de l'innovation vue comme un processus est maintenant utilisé en
France par différents chercheurs dans le domaine agricole, qu'ils relèvent des sciences biotechniques, comme l'agronomie ou la médecine vétérinaire (Bouyer et al 2011a et b, Duru et al 2011, Ingrand et al 2012) ou des sciences de gestion (Chia 2004). Leurs pratiques de recherche, visant à apporter une contribution dans les processus d'innovation, s'inscrivent donc dans cette perspective. Selon Meynard et al (2006), les chercheurs peuvent ainsi : $i$ ) être à l'origine de l'innovation en proposant une invention ; ii) proposer aux acteurs des outils et méthodes pour innover par eux-mêmes ou pour évaluer l'intérêt potentiel, pour leur propre situation, d'innovations exogènes ; iii) identifier, analyser, améliorer et promouvoir des innovations conçues par des acteurs de terrain; $i v$ ) aider à anticiper les effets économiques, sociaux et environnementaux des innovations, les conditions de leur adoption, et leurs conséquences à différentes échelles.

Dans le monde de l'élevage, les acteurs participant au processus d'innovation sont multiples et divers, comme le présentent Meynard et Dourmad (2014) dans le premier article de ce numéro spécial. Pour notre part, nous illustrons cette idée par des exemples d'innovations " en ferme », c'est à dire quand les éleveurs contribuent eux aussi à l'émergence du processus.

Nous tenterons tout d'abord de faire une synthèse de travaux génériques afin de préciser ce qu'est l'innovation et ce qui est en jeu dans le processus d'inno- vation, pour proposer finalement une classification des innovations. Partant de travaux associés à différents cas d'étude et différentes orientations disciplinaires, nous montrerons comment ces notions et théories sont mobilisées dans le champ de l'agriculture et nous les illustrerons par des exemples d'innovations en ferme dans le domaine de l'élevage.

\section{1 / Qu'est-ce que l'innovation et quelles en sont les compo- santes?}

\section{1 / La nouveauté : composante essentielle de l'innovation}

Le concept de « nouveauté » fait partie intégrante de l'innovation. Selon Frank (1995a) et Deltour (2000), l'innovation peut prendre la forme d'une technique, d'un matériel, d'une idée, etc., possédant l'attribut de nouveauté. Cependant, cette notion est toujours relative à quelque chose qui existe déjà. Dans ce sens, Schroeder et al (1989) explicitent le caractère non absolu de l'innovation : " une idée est innovatrice à partir du moment où elle est perçue comme nouvelle par les personnes impliquées, même si, pour d'autres personnes, elle semble être une imitation de quelque chose qui existe déjà ailleurs ». Ainsi est qualifié de « nouveau » ce qui n'est pas utilisé de façon routinière dans une région, qui va à l'encontre des pratiques locales habituelles. Le qualificatif de « nouveau » 
est donc éphémère. Il est relatif à celui qui en parle et à son référentiel : contexte local, social, territorial, etc. au moment où la nouveauté est constatée.

En agriculture, l'apparition de nouveautés est un phénomène courant. Les agriculteurs mobilisent le pool de ressources de leur exploitation (Darnhofer et al 2010) pour créer de la nouveauté (Goulet et al 2008). L'innovation est considérée comme l'appropriation d'une nouveauté, du point de vue d'un agriculteur, intégrée dans son système, c'est à dire incluse de manière significative dans son itinéraire technique (Bal et al 2003). Par exemple, dans la filière viticole, la plantation de nouveaux cépages en Languedoc, même s'ils étaient déjà employés dans d'autres régions, représente une " innovation produit », permettant à des caves d'améliorer et de diversifier leur gamme (Montaigne et Cadot 2006). En élevage, Lasseur (2005) définit des « systèmes d'élevages locaux », faisant appel à des normes et des pratiques, partagées entre les éleveurs d'un groupe professionnel local (Darré 1996), et dont toute modification pourra être perçue comme une innovation à l'échelle locale.

Dans le Parc Naturel Régional (PNR) du Pilat dans les années 2000, une douzaine d'éleveurs de bovins laitiers se sont convertis à l'Agriculture Biologique (AB), processus qui, localement, pouvait être caractérisé comme une innovation. En effet, à cette période et dans cette région, aucun éleveur ne conduisait son système de production selon les principes de l'AB. Il s'agissait donc pour les éleveurs de reconcevoir leurs systèmes de manière à, comme ils le décrivent eux-mêmes, « produire sans ammonitrate ", avec tous les changements de repères et de pratiques que cela implique (Gouttenoire et al 2013).

Le passage à la monotraite en élevage bovin laitier peut également être considéré comme une innovation, en tant que nouveauté dans un contexte donné. Les fréquences de traite appliquées à une vache sont diverses à l'échelle mondiale, de plusieurs fois par jour dans les systèmes avec robot de traite (Billon et Pomiès 2006), à une fois par jour, voire moins dans les systèmes avec partage du lait entre le veau et la famille (Milleville 1991). Historiquement, la traite deux fois par jour s'est imposée comme la norme dans les systèmes laitiers spécialisés avec traite mécanique. Cependant, le passage à la monotraite dans ce type de système est une solution déclarée comme courante en Nouvelle-Zélande (Davis et al 1999), mise en œuvre comme i) outil de pilotage de l'état des vaches (équilibre énergétique), en début ou en fin de lactation, voire sur toute la lacta- tion, et pour améliorer la productivité du travail ou permettre le partage de la salle de traite entre exploitations. En France, cette pratique est plus récente.

Au-delà donc de la nouveauté pour un individu, c'est plus le caractère nouveau par rapport au un réseau social qui est mis en avant.

\section{2 / L'innovation : à distinguer d'autres concepts}

Le concept d'innovation ne saurait se réduire à la simple idée de nouveauté, en particulier parce qu'il induit une notion de dynamique, de processus. $\mathrm{Ce}$ processus d'innovation est souvent associé à trois autres processus, qui pour autant ne le recouvrent pas, à savoir adaptation, changement et invention. Dagenais et al (2005) insistent ainsi sur la nécessité de distinguer les différents termes « innovation, invention, adaptation et changement ", même si les définitions de chacun d'entre eux sont proches.

\section{a) Innovation et invention}

De nombreuses citations, reliant l'innovation à l'invention, sont évoquées dans la littérature. L'invention correspond à la découverte d'un principe, l'action de trouver et son résultat, alors que l'innovation est plutôt l'appropriation de ce principe par le corps social. " L'inventeur peut être un génie dénué de sens pratique mais pas l'innovateur qui se charge de trouver [...] un usage à la découverte » (Alter 2010). Dans le langage courant, il n'est pas rare que les deux termes soient employés indistinctement l'un de l'autre. En effet, ils contiennent tous les deux les notions de nouveauté. Cependant, une invention devient une innovation lorsqu'elle rentre dans le domaine social, c'est-à-dire quand elle rencontre des utilisateurs. L'étape du processus qui correspond au développement de l'invention est comparée à la " maturation », et le passage final à l'innovation est baptisé « socialisation de l'invention » (Alter 2010).

\section{b) Innovation et adaptation}

En général, l'adaptation fait suite à un problème et se définit comme une action en réaction à un événement. En ce sens, elle est plutôt subie. Par ailleurs, les individus adaptent leurs connaissances déjà acquises et la notion de nouveauté n'intervient pas nécessairement. Ainsi, les solutions mises en œuvre par les éleveurs pour s'adapter à des périodes de sécheresse, relèvent de la mise en œuvre de pratiques courantes localement, comme l'achat de foin pour compenser la perte de production d'herbe, suite à la sécheresse de 2003 dans le Sud-Est de la France (Moulin et al 2009), ou la vente d'animaux pour réduire les besoins alimentaires du troupeau, dans les systèmes ovins au Maghreb (Bourbouze 2006). L'innovation ne fait pas toujours suite à une complication et s'inscrit dans le cadre d'un processus volontaire. Cependant, comme le signalent Goulet et al (2008), " les individus commencent par s'adapter et finissent par innover ». Par exemple, la répétition d'années sèches, amenant le montage d'opérations d'approvisionnement en fourrages organisé par une " cellule sécheresse » départementale, a conduit des groupes d'éleveurs à contractualiser avec des maïsiculteurs. En cas de sécheresse, les maïsiculteurs récoltent une partie de leur production en ensilage pour les éleveurs, plutôt qu'en grain. Le contrat prévoit les règles de fixation du prix, pour compenser le manque à gagner pour les maïsiculteurs. L'adaptation à la sécheresse a conduit ici à une innovation organisationnelle sur la base de techniques courantes (Delerce et al 2011).

\section{c) Innovation et changement}

Selon Alter (2010), le changement est la transformation d'un ou plusieurs éléments du système et correspond à la comparaison de deux états (initial et final). Toutefois, les entreprises actuelles sont en mouvement perpétuel et les changements sont permanents, l'état final n'est donc jamais clairement identifié (Rammel et Van den Bergh 2003, Alter 2010). Le changement est ainsi considéré comme un processus de modification de l'état initial. Les changements de pratiques sont des phénomènes courants, voire récurrents en agriculture. Les agriculteurs testent des alternatives le temps d'un projet, rendant le changement réversible. Ce type de changement est qualifié d'innovation par Bentz et Gretz (2002), tandis que pour Le Bail et Meynard (2003), cela n'en fait pas pour autant une innovation. Les recouvrements de définitions ainsi mis en évidence selon les auteurs montrent que la limite entre innovation et changement de pratiques est délicate à définir.

Cros (1997), s'intéressant à l'innovation dans le domaine de la formation et de l'éducation, désigne l'innovation comme un changement intentionnel et délibéré. D'après la définition de West et Altink (1996 in Cros 1997), l'innovation contient également une intention d'amélioration. Ainsi, des changements imposés ne seraient pas à considérer comme des innovations. Par exemple, l'identification électronique des ovins est présentée dans une fiche à destination des éleveurs comme une obligation européenne permettant l'amélioration de la gestion des crises sanitaires afin de 
Figure 1. Séquences du processus de développement de l'innovation selon la théorie de Schumpeter (1935).

Selon Schumpeter, la trajectoire de l'innovation débute de l'élaboration d'une nouvelle idée à son utilisation. Sa théorie se découpe ainsi en trois temps : 1/ la combinaison de nouveaux facteurs par des individus " marginaux " (à l'encontre du circuit économique dominant) : c'est donc l'apparition d'une invention ; $2 /$ lorsque la rentabilité économique est démontrée, des imitateurs récupèrent et adaptent ces inventions ce qui engendre des " grappes » : la situation est alors instable ; 3 / de nouvelles règles sont élaborées : la situation redevient normale, l'innovation est réussie.

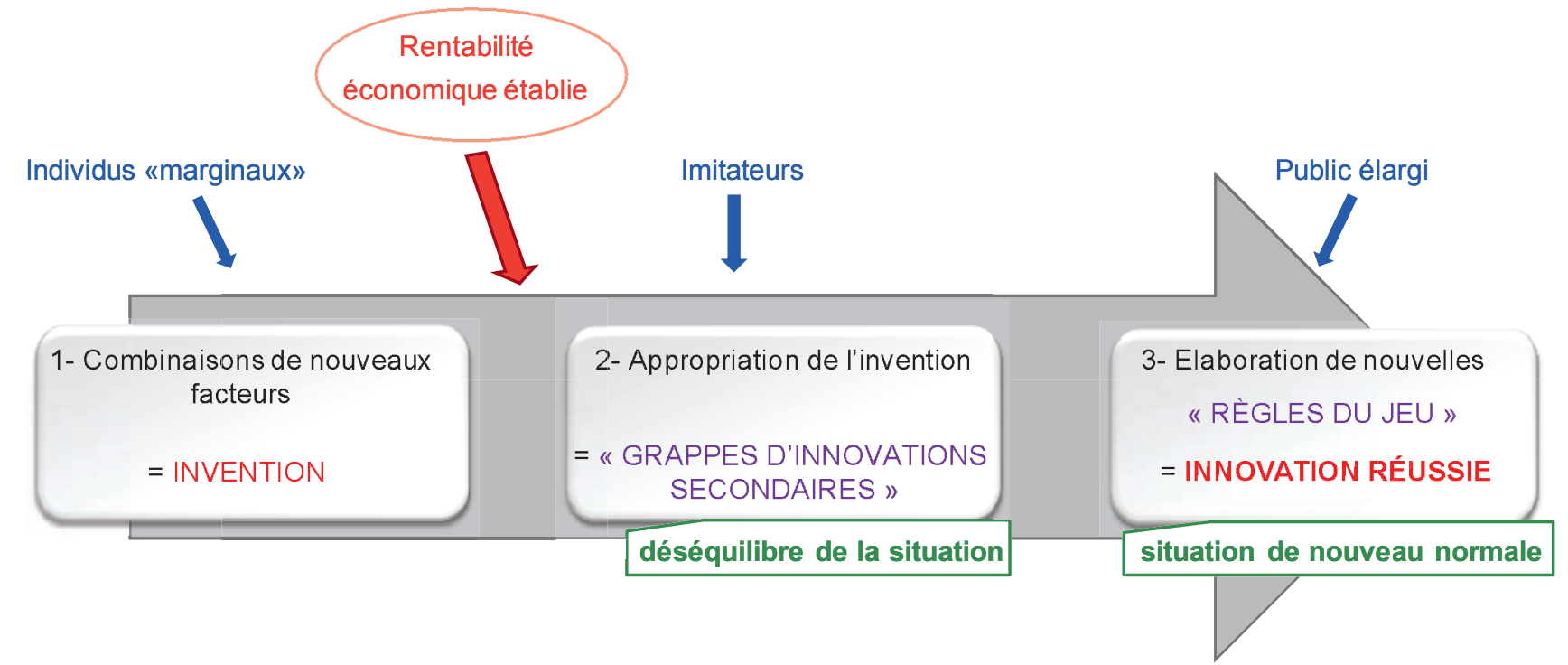

préserver la santé des animaux et des consommateurs (Institut de l'Elevage, non daté). Selon la définition de West et Altink (1996), l'adoption de puces électroniques, reposant sur une obligation, est donc bien un changement en ferme, mais ne serait pas une innovation. La mise en œuvre en fermes de dispositifs techniques complémentaires (avec notamment des lecteurs des puces RFID, fixes ou mobiles), pour faciliter des opérations de conduite (tri) ou individualiser des pratiques, représenterait en revanche des innovations en ferme (Marguin et Balvay 2009) (Bocquier et al 2014, ce numéro), relevant d'une volonté des éleveurs.

Une innovation n'est donc repérée que sur le long terme, quand elle s'est intégrée dans le tissu social. Elle peut être interprétée comme une adaptation, un changement ou une invention, mais la réciproque n'est pas toujours vraie. En effet, toute adaptation, tout changement ou toute invention n'est pas une innovation.

\section{3 / Le processus d'innovation est séquentiel et interactif}

Au début du $X^{\text {ème }}$ siècle, alors que l'innovation avait jusque-là une connotation négative, considérée comme néfaste pour l'évolution des sociétés (Cros 1997), l'économie politique est à l'origine d'une vision positive de l'innovation. L'économiste Schumpeter a ainsi proposé la première théorie économique sur l'innovation. Ses travaux sont considérés comme la base fondatrice de la philosophie de l'innovation (Dalglish et al 2002,
Alter 2010). Schumpeter (1935) distingue 3 séquences dans le processus aboutissant à l'adoption de l'innovation avec l'apparition d'une nouvelle norme (figure 1).

- La première séquence correspond à l'apparition de nouvelles façons de faire par quelques individus en marge du circuit économique dominant pour sortir de la routine. Ces nouvelles façons de faire sont souvent perçues comme risquées.

- La deuxième séquence correspond à une situation où, dès lors que la rentabilité économique de ces nouvelles façons de faire est démontrée, des imitateurs les récupèrent, les généralisent, les adaptent, développant ainsi des " grappes d'innovations secondaires ».

- Enfin, la troisième séquence est l'établissement de nouvelles règles qui confirment l'adoption de l'innovation avec l'apparition d'une nouvelle norme. De nouvelles règles du jeu sont établies et la situation redevient à l'équilibre.

Selon Schumpeter (1935), l'invention et la mise en pratique de l'innovation correspondante sont décalées dans le temps, et économiquement et sociologiquement dissociées. Ce type de modèle laisse apparaître l'innovation comme un processus linéaire et individuel. On parle de modèle « séquentiel ». Il considère que l'aspect social n'intervient qu'après l'adoption de l'innovation. Pour cela, il est souvent jugé simplificateur de la réalité des différents acteurs en interaction. Pour d'autres auteurs, le caractère cognitif du processus d'inno- vation est déterminé par une accumulation des connaissances et des compétences au sein des organisations (phénomène d'apprentissage) (Nonaka et Takeuchi 1997). Depuis le début du XX ${ }^{\text {ème }}$ siècle, les sociologues ont mis en évidence la contribution du processus social dans le développement du processus d'innovation. Ainsi, l'innovation ne concerne pas un acteur ou une organisation, mais plutôt les interactions entre les acteurs qui en sont constitutifs (Callon et Latour 1989), et le processus d'innovation correspond à des séquences temporelles d'évènements dans un contexte institutionnel (Van de Ven et Poole 1995). On parle ici de modèle « interactif».

Parmi les modèles « interactifs », le modèle le plus souvent retenu est celui d'Akrich et al (1988). Il est baptisé le « modèle tourbillonnaire » (tableau 1). Ce modèle prend en compte le retour possible à une situation normale de laquelle peut redémarrer un cycle d'innovation.

L'analyse de l'innovation en agriculture a fortement été orientée par le contexte socio-économique. Deux types de travaux se sont distingués au cours du temps : ceux portant sur les effets de l'innovation, en général en termes d'efficacité de la production, et ceux, plus récents, portant sur les processus d'innovation, abordant plus spécifiquement la co-construction d'objets techniques et le contexte de leur diffusion (Lemarié et Joly 2000). Ce second cas, plus actuel, voit donc apparaitre progressivement un modèle multipolaire où l'innovation résulte de l'interaction entre des acteurs hétérogènes. 
Tableau 1. Comparaison du modèle classique de diffusion et du modèle tourbillonnaire (selon Akrich et al 1988).

\begin{tabular}{|c|c|}
\hline Modèle classique de la diffusion & Modèle tourbillonnaire \\
\hline $\begin{array}{l}\text { - Modèle de la diffusion : " l'innovation s'impose et se } \\
\text { répand d'elle-même par contagion grâce à ses } \\
\text { propriétés intrinsèques » } \\
\text { - C'est aux utilisateurs de l'innovation de s'adapter soit } \\
\text { de force soit par ennui } \\
\text { - L'innovation et son environnement socio-économique } \\
\text { sont irrémédiablement séparés } \\
\text { - Le travail d'élaboration est restreint au cercle limité } \\
\text { des concepteurs responsables du projet : pas de } \\
\text { dimension collective de l'innovation } \\
\text { - La majorité des acteurs est passive }\end{array}$ & $\begin{array}{l}\text { - Modèle de l'intéressement: « le destin de l'innovation } \\
\text { dépend de la participation active de tous ceux qui sont } \\
\text { décidés à la faire avancer » } \\
\text { - De nombreux liens réunissent l'« objet » à tous ceux qui } \\
\text { le manipulent } \\
\text { - Le processus de conception intègre dès le départ tous } \\
\text { les acteurs du réseau } \\
\text { - Le processus d'innovation est assimilé à une recherche } \\
\text { continue de compromis et d'adaptations } \\
\text { - Le mouvement d'adoption est un mouvement } \\
\text { d'adaptations } \\
\text { - La majorité des acteurs est active }\end{array}$ \\
\hline
\end{tabular}

\section{2 / Le processus d'innovation en ferme}

\section{1 / Les sources de l'innovation}

Depuis des siècles, au-delà des innovations « scientifiques », les agriculteurs innovent par eux-mêmes. Ces pratiques sont qualifiées d' « innovations paysannes : techniques ou pratiques expérimentées ou adoptées par des agriculteurs sans faire suite à un transfert d'une entreprise, sinon un paysan proche » (Sabourin et al 2004). Les travaux sur l'innovation en agriculture mettent ainsi souvent en avant la distinction entre des innovations de source exogène, idées issues de la recherche publique et privée, ou globalement de l'appareil de recherchedéveloppement, et des innovations de source endogène, issues du « milieu », en référence au monde des agriculteurs, des paysans (Lefort 1988). Le fait que la source de l'innovation soit exogène est parfois mis en avant comme facteur explicatif de l'échec d'appropriation d'une innovation. Landais et Lhoste (1990) défendent ainsi l'idée que c'est pour cette raison que le «modèle technique » de l'association agricultureélevage, porté par la recherche agronomique, n'est jamais «passé » en milieu rural en Afrique intertropicale. Ils remettent en cause les raisonnements techniques et les hypothèses sur lesquelles ce modèle s'appuie pour expliquer l'échec de sa diffusion dans les systèmes de production agricole d'Afrique de l'Ouest.

Dans une étude récente (projet Casdar « Systèmes d'élevage allaitants innovants; Salinov », Ingrand et al 2012, Pottier et al 2014), des enquêtes ont été menées chez 49 éleveurs bovins ou ovins jugés par leurs pairs « différents », « atypiques », « pas dans la norme ». Au total, 150 pratiques innovantes ont été recensées, 64 dans les 24 élevages bovins et 86 dans les 25 élevages ovins. Les pratiques innovantes ainsi identifiées dans les élevages (tableau 2) concernent d'abord les pratiques d'élevage " de base » (alimentation et reproduction), ainsi que les structures (bâtiments et équipements), ceci dans les deux productions (bovine et ovine). Les différences entre espèces concernent les pratiques de commercialisation (vente directe, diversification de gamme), plus fréquemment concernées en élevage bovin ; le travail, également plus représenté en élevage bovin ; et surtout les pratiques de pâturage, qui, en élevage ovin cette fois, concernent $10 \%$ des pratiques innovantes recensées, contre $2 \%$ seulement en élevage bovin. A noter que la diversification des activités sur la ferme, incluant des activités hors élevage, voire non agricoles, représente de 5 à $6 \%$ des changements recensés.

Tableau 2. Domaines d'élevage pour les pratiques innovantes recensées dans 49 fermes (64 dans les 24 élevages bovins et 86 dans les 25 élevages ovins).

\begin{tabular}{|l|c|c|}
\hline Domaine d'élevage & Bovins (\%) & Ovins (\%) \\
\hline Alimentation & 20 & 14 \\
\hline Reproduction & 13 & 15 \\
\hline Commercialisation & 6 & 2 \\
\hline Conduite du troupeau & 5 & 6 \\
\hline Génétique & 3 & 5 \\
\hline Santé & 2 & 3 \\
\hline Equipement & 14 & 22 \\
\hline Bâtiments & 11 & 9 \\
\hline Travail du sol & 5 & 5 \\
\hline Récolte & 3 & - \\
\hline Conduite des cultures & 2 & 10 \\
\hline Pâturage & 2 & 2 \\
\hline Travail & 6 & - \\
\hline Energie & 3 & 6 \\
\hline Diversification & 5 & \\
\hline
\end{tabular}

La pratique montre que la majorité des innovations en agriculture ont des sources mixtes, c'est-à-dire à la fois endogènes et exogènes (Bal et al 2003). Une référence extérieure est recombinée et réinterprétée en interne par les agriculteurs, en fonction des finalités propres de leur système (Bentz et Gretz 2002). « D'origines endogènes ou exogènes [...], c'est souvent une combinaison des deux qui s'opère » (Pichot et Faure 2008).

Une autre distinction porte sur le réseau à l'origine de l'innovation. L'origine peut être individuelle : l'innovateur a travaillé seul, de façon isolée sans influence directe de son entourage. Le plus souvent, les innovateurs ont travaillé collectivement, sous l'influence de leur entourage (Sulpice et al 2006). Comme souligné par Lemarié et Joly (2000), le secteur agricole se caractérise par un 
nombre très important d'organisations et d'institutions, ce qui favorise particulièrement un processus d'innovation collectif au sein d'un système social en interaction.

\section{2 / Echanges d'idées entre acteurs, dispositifs facilitateurs}

L'innovation est donc un processus collectif, qui repose sur la mobilisation d'acteurs aux rationalités variées, souvent antagonistes. Pour comprendre ce processus et favoriser les échanges entre acteurs, des dispositifs spécifiques doivent être mis en place, pour mieux comprendre les rapports formels et informels, les relations de pouvoir et de conflits, mais surtout pour tenir compte du caractère contextuel et particulier du secteur.

Au-delà de l'idée d'origine, les travaux sur l'innovation en ferme mettent en évidence un phénomène d'adaptation, d'appropriation de l'innovation. Les techniques agricoles doivent le plus souvent être « ajustées, modifiées ou harmonisées (ou même abandonnées), selon les conditions agro-écologiques et économiques qui caractérisent chaque milieu » (Lefort 1988). La technique peut ainsi être reprise, adaptée, transformée à l'échelle locale par une diversité d'acteurs (Leeuwis et Van Den Ban 2004). Un groupe social s'approprie ainsi une nouveauté et la transforme dans le temps en tenant compte de différentes dimensions (techniques, culturelles, économiques, organisationnelles) et des savoir-faire locaux (Chia 2004). Ameur et al (2013), évoquent ainsi le processus de «domestication » de l'innovation, en analysant le cas de l'utilisation de l'irrigation par goutte à goutte dans les petites exploitations au Maroc. Le concept de domestication, appliquée à la technologie, permet de considérer que les acteurs et leurs relations à la technique changent aussi au cours du processus d'innovation : " construction d'un ensemble de pratiques, construction du sens de l'artefact, processus cognitifs liés à l'apprentissage » (Sørensen 2006).

Les démarches participatives associant diagnostics, refonte de système, expérimentations en fermes et évaluation facilitent les échanges entre acteurs autour des différentes phases du processus d'innovation (Dogliotti et al 2014). Un projet de recherche-action a été initié dans cette perspective au Cambodge pour améliorer la compétitivité de la filière porcine (Binh 2010). Le projet visait l'accès des petits producteurs à des marchés à haute valeur ajoutée en jouant sur une amélioration de la qualité de la viande par le déploiement de techniques innovantes (notamment en matière d'alimentation). Une démarche collective, notamment dans le cadre de groupements de producteurs, a rendu possible une relation plus équitable entre tous les acteurs de la filière et de meilleurs revenus ont ainsi été assurés aux éleveurs familiaux. En Afrique, Bouyer et al (2011a et b) ont décrit le processus d'innovation autour d'un dispositif technique de lutte sanitaire contre les glossines, vecteur des trypanosomes. Partant de l'idée que l'application restreinte d'insecticides est une méthode intéressante pour contrôler les populations de glossines, des expérimentations ont été menées en station pour en mesurer l'efficacité, suivies d'une phase d'expérimentation en milieu paysan. Deux pédiluves ont été construits dans des villages proches de la station expérimentale, dans le cadre d'une recherche-action menée avec deux groupes de paysans. Le travail a été conduit pendant quatre ans, permettant d'améliorer " chemin faisant » le pédiluve et les équipements associés. Un paquet technique (construction du pédiluve, association de matières actives acaricides et insecticides, rythme d'utilisation selon les saisons...) a ainsi été construit grâce aux interactions entre chercheurs et éleveurs. Exogène au départ, cette innovation peut ainsi être considérée comme mixte (Bouyer et al 2011a). Dans une phase ultérieure, ce paquet technique a été vulgarisé, à partir de fiches techniques, d'ateliers de présentation de la méthode avec des visites sur le terrain et l'organisation de débats au sein des organisations d'éleveurs. Une étude de l'appropriation de l'innovation auprès de groupes d'éleveurs ayant adopté le pédiluve (Bouyer et al 2011b) montre que le " paquet technique » a encore évolué durant cette phase. Par exemple, les groupes d'éleveurs ont mis en place des configurations variées de parcs d'attente pour accéder au pédiluve. Ces configurations sont associées à des efficacités très différentes (proportion d'animaux dans le troupeau passant effectivement par le pédiluve au retour du pâturage), et le parc d'attente constitue une innovation en soi.

Cet exemple montre bien l'évolution possible et même souhaitable de l'idée initiale, son enrichissement par des dispositifs techniques qui l'accompagnent et qui vont permettre finalement la maitrise de l'innovation et son appropriation. Au cours de ce processus, la transformation de l'innovation peut être telle que son usage peut s'avérer très différent de celui prévu à son origine. Duru et al (2011) montrent ainsi comment un outil (Herb'âge), destiné simplement au départ à transformer des dates de récolte en sommes de température, a été utilisé par des conseillers pour réaliser des cartographies à l'échelle de petites régions agricoles. Ceci leur permet d'anticiper les dates clés de conduite des systèmes d'alimentation et de mettre en place des messages d'alerte par voie de presse ou messagerie électronique. Pour ce dispositif d'alerte, les conseillers ont ainsi également simplifié la méthode de qualification des prairies proposée par les chercheurs.

Mohr (1982) suggère d'étudier les processus d'innovation en fonction de contextes organisationnels et technologiques spécifiques. Le secteur agricole est particulièrement soumis aux pressions sociétales. Ainsi, si le concept actuel d'innovation en agriculture contient implicitement l'idée d'offrir un bénéfice aussi bien économique que social pour l'agriculteur, la prise en compte de l'impact des innovations sur l'environnement et le bien-être animal est devenu incontournable depuis les années 1980. Ainsi, la spécificité des systèmes d'élevage où les processus de production portent sur des animaux, dont le statut est particulier et a beaucoup changé ces dernières années dans les sociétés modernes, y compris pour les animaux de rente, est un facteur essentiel à prendre en compte dans l'analyse des processus d'innovation. Cela est illustré dans l'article d'Hostiou et al (2014) dans ce numéro, consacré à l'élevage de précision.

\section{3 / Dynamique du processus d'innovation au sein d'un groupe social}

La diffusion d'une innovation est un processus qui ne se limite pas à la somme des adoptions individuelles puisqu'il peut nécessiter l'intervention d'autres acteurs que les adoptants potentiels (conseillers, prescripteurs, fournisseurs, agents de la filière...), et qu'il est influencé par les caractéristiques du système social dans lequel il se déroule.

a) L'adoption d'une innovation par un individu dépend de son degré d'implication au sein d'un groupe social

En considérant le groupe social comme le socle de diffusion de l'innovation, les sociologues apportent une vision complémentaire à celle proposée par Schumpeter (1935). Alter (2010), explique ainsi que "l'innovation est toujours une histoire, celle d'un processus », important à comprendre d'un point de vue social, c'està-dire sans le limiter à la seule logique économique. En effet, une invention n'est jamais suivie mécaniquement par son adoption, laquelle dépend des besoins, des attentes, des points de vue (attirance, méfiance, rejet), vis-à-vis de cette innovation. Une innovation peut donc se développer avec succès dans un certain contexte, mais pas dans un autre (Rammel et Van den Bergh 2003).

Les dynamiques du processus d'innovation décrites par les économistes ou 
Figure 2. La diffusion des innovations selon Rogers (1962), avec l'identification de catégories successives des " adoptants " parmi la population des utilisateurs potentiels.

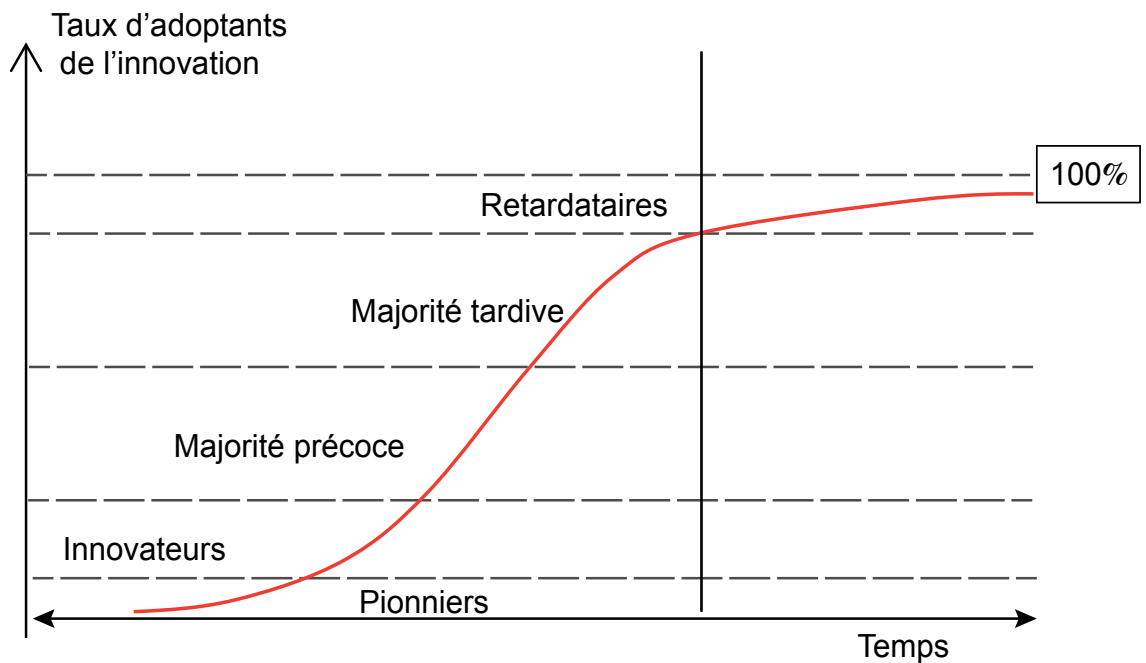

les sociologues ont été intégrées dans un modèle générique de diffusion, avec la possibilité de calculer un taux d'adoption de l'innovation. Ce modèle a été conçu par Rogers (1962), professeur en sociologie rurale ; il décrit les dynamiques de diffusion de l'innovation dans le milieu agricole. Cinq catégories d'individus adoptants sont décrites, le long d'une courbe sigmoïde, selon l'évolution du taux d'adoption de l'innovation (figure 2) : les « pionniers », puis les « adopteurs précoces », puis la « majorité précoce », puis la « majorité tardive » et enfin les " retardataires ». Les « pionniers » sont représentés par quelques individus atypiques, prêts à prendre des risques. Les « suiveurs » ou " adopteurs précoces », s'associent rapidement à la nouveauté ; l'adoption se fait avec lucidité et adaptations. Pour la « majorité précoce », la trajectoire de la courbe s'oriente alors vers le haut après

Figure 3. L'approche multi-niveaux des transitions permises au sein des régimes sociotechniques dominants, grâce aux innovations issus de niches et à l'évolution du contexte (selon Geels 2011).

\section{Consolidation des pratiques}

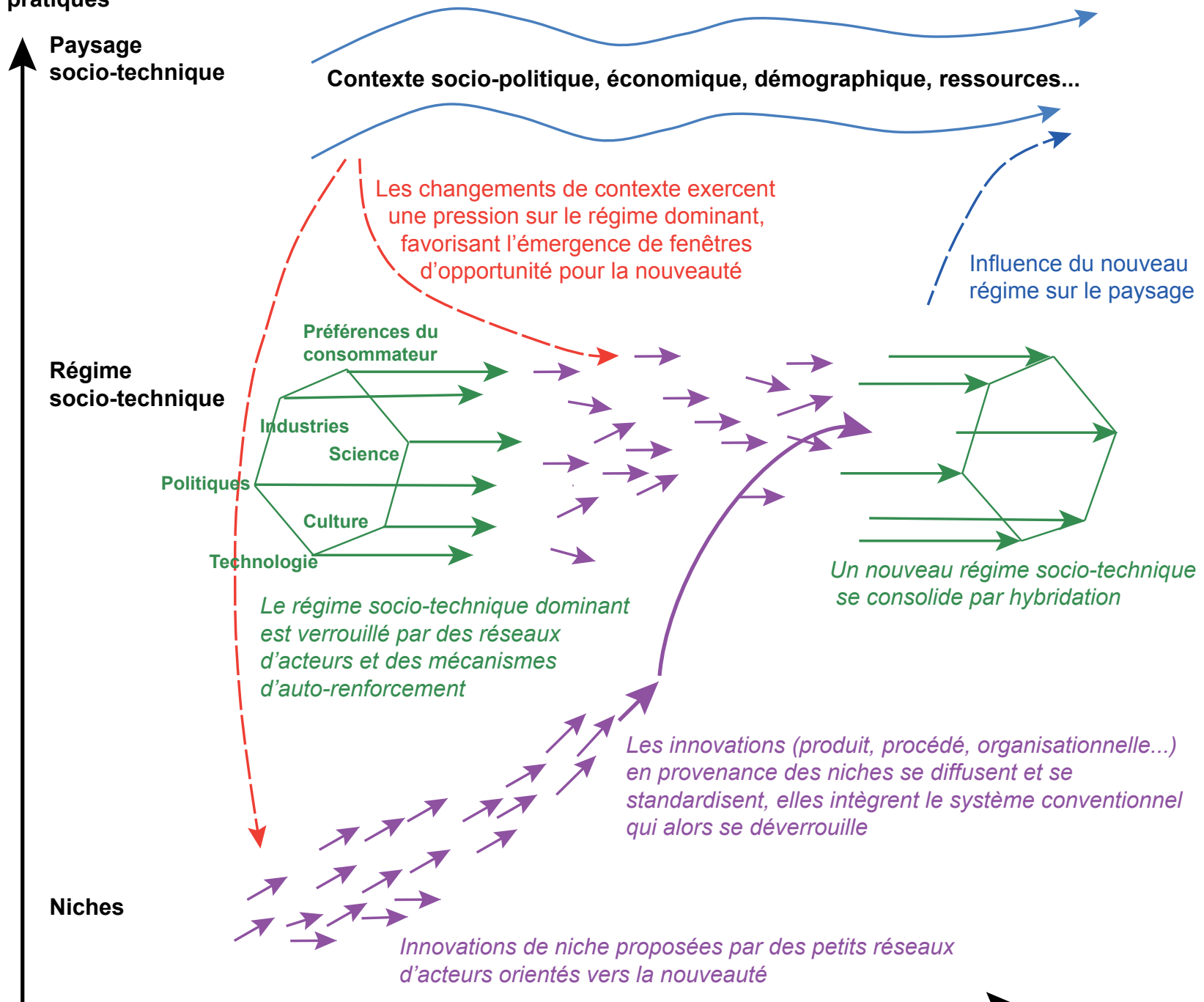
d'acteurs orientés vers la nouveauté 
un temps variable, quand la majorité de la population soumise à l'innovation l'a adoptée. Progressivement, le nombre d'individus concernés par l'innovation diminue, la population d'utilisateurs potentiels n'étant pas extensible. La quatrième catégorie est « la majorité tardive » qui considère l'innovation avec scepticisme. Enfin, les « retardataires » constituent le plateau de la sigmoïde : ils ont tendance à se concentrer sur la tradition et ont une aversion assez marquée au changement. Ce modèle aboutit à un taux d'adoption de $100 \%$.

Ce modèle de la diffusion est cependant discuté. En effet, selon Frank (1995b), la typologie de Rogers (1962) a été construite sur la base implicite que l'innovation est un comportement souhaitable. Or, selon ce dernier auteur, les deux dernières classes d'adoptants peuvent avoir de très bonnes raisons de ne pas adopter une innovation (Landais et Deffontaines 1988). Une même innovation ne présente donc pas le même intérêt pour tous les agriculteurs et elle doit être adaptée au contexte dans lequel elle est implantée (Jullian 1989). Le modèle de diffusion de l'innovation de Rogers (1962) ne prend pas en compte les exploitants qui n'acceptent pas l'innovation (taux d'adoption final de 100\%). En d'autres termes, une innovation peut donc être refusée par une ou des catégories d'agriculteurs alors même qu'elle devient une nouvelle norme pour d'autres qui l'ont totalement adoptée.

La théorie de la transition dans les systèmes sociotechniques (Elzen et al 2004), apporte un autre éclairage sur le processus d'innovation. Cette approche multiniveau (Geels 2011) constitue un cadre d'analyse heuristique qui permet d'expliquer comment l'impact des inflexions contemporaines peut se traduire par l'émergence de nouvelles niches de production et de consommation, à partir desquelles le système de production conventionnel peut s'hybrider et transiter vers un nouveau système (i.e. vers un nouveau régime sociotechnique). La conception de ces niches ouvre une perspective de déverrouillage des systèmes de production via une forme de transition, qui s'apparente à un mécanisme de diffusion progressive de l'innovation au sein du système conventionnel.

Selon ce modèle, l'innovation se fait d'abord dans des niches (figure 3). Elle peut ensuite être transposée progressivement au niveau du régime sociotechnique, qui incarne le mode dominant d'accomplissement d'une fonction sociale donnée (par exemple la production agricole, le logement, la communication...) à un moment donné, si les modifications de son environnement (le " paysage ", composé des "mouvements de fond» comme les grandes tendances démographiques et géopolitiques, les structures socioculturelles profondes, les évolutions économiques et environnementales) lui deviennent favorables. Ainsi, dans ce modèle, il n'est plus question d'individus favorables ou réfractaires aux innovations, mais de mouvements de fond propices ou non à la diffusion des innovations apparues dans les niches.

\section{b) Comprendre les facteurs favorables ou défavorables au processus d'innovation en ferme}

Le processus d'innovation n'est pas toujours aisé et les acteurs sensés le promouvoir doivent parfois surmonter des résistances. Les auteurs s'accordent généralement sur deux types de résistances, un facteur social (ou humain) et un facteur financier (ou capital), mais les interprètent différemment. En effet, l'innovation induit un manque de stabilité de la situation et une incertitude qui limite la rationalité du gestionnaire. Enfin, le manque de repères sociaux et d'informations crée des oppositions entre partenaires de l'innovation. Ces différents facteurs ralentissent ou inhibent le processus d'innovation (Alter 2010).

Plusieurs auteurs soulignent le rôle des facteurs socioéconomiques dans le processus d'innovation en agriculture (pour une revue voir Barruentos-fuentes et Berg 2013). Un agriculteur doit être convaincu des avantages socioéconomiques liés à l'adoption d'une innovation. Il doit lui trouver une « utilité » c'est-à-dire une rentabilité, une utilité pratique et/ou une satisfaction personnelle (Franck 1995b). Toutefois, dans le cas où un événement critique justifie l'attrait pour une innovation, le facteur déterminant reste économique, en particulier le rapport « coûts engagés » pour accéder à l'innovation sur les «bénéfices utiles » engendrés par l'innovation.

Le choix d'adopter ou non une innovation suivrait donc une rationalité en lien avec le contexte et les besoins socioéconomiques d'une exploitation. Bentz et Gretz (2002) rejoignent cette vision en précisant que l'innovation n'est pas toujours souhaitée pour augmenter la productivité économique d'une exploitation mais bien pour permettre « un gain de revenu ou de productivité du travail », ou une meilleure valorisation des ressources (écosystèmes, cultures, maind'œuvre), ou encore une « réduction des risques et une plus grande sécurisation de l'économie familiale ». Quelques études seulement (Lynne et al 1988, Lynne 1995, Willock et al 1999) ont exploré les motivations et les attitudes des adoptants et non-adoptants, et les ont associées au processus d'adoption en tant que tel.
Le bénéfice économique n'est pas tout. Certaines innovations ne contribuent pas significativement à l'amélioration des bénéfices économiques, mais affectent aussi les bénéfices sociaux et environnementaux (Barruentes-Fuentes et Berg 2013). Matanmi (1994) propose aussi d'autres pistes comme la compatibilité de l'innovation avec les valeurs, les expériences passées et les besoins des utilisateurs. Une innovation qui n'est pas compatible avec les caractéristiques de la société ne sera pas acceptée dans cette société. Par exemple, l'étude de Diallo (1994) a montré que l'échec du développement de nouveaux produits agricoles transformés était principalement dû au fait que les attentes des consommateurs n'avaient pas été prises en compte (ils n'avaient pas l'habitude de consommer les produits développés). Par ailleurs, une étude de Frank (1997), a montré que des éleveurs bovins préféraient maintenir leur style de vie en accord avec leur société, plutôt que d'adopter des innovations pourtant avantageuses économiquement.

Enfin, Dockès et al (2004) évoquent le manque d'apport de connaissances et d'informations comme limite aux changements, car cet apport est souvent indispensable dans la prise de décisions des éleveurs. Les agriculteurs ayant une vision claire des avantages d'une innovation sont plus enclins à l'intégrer dans leur système, puisqu'ils ont une meilleure perception de ce qu'elle peut leur apporter par rapport à leurs propres objectifs.

Ces définitions et ces illustrations nous permettent de donner une architecture au processus d'innovation, mais ne permettent pas de rendre compte de l'intensité et de l'impact de l'innovation en ferme.

\section{4 / L'adoption d'une innovation induit des changements qui peu- vent influencer le processus de diffusion}

A partir de travaux sur l'innovation dans différents secteurs, nous proposons deux clés d'analyse, l'une basée sur le degré de changement induit et l'autre sur les objets concernés par l'innovation. Nous nous appuierons sur des exemples dans le secteur de l'élevage pour illustrer chacune d'elles.

\section{a) Les formes d'innovation, selon le degré de changement induit}

Trois types d'innovations peuvent être distingués selon le degré de changement induit dans le système considéré (Willinger et Zuscovitch 1993, Giget 2007) :

Innovation d'optimisation, incrémentale, de continuité : elle consiste en une mise à niveau de l'existant. C'est une amélioration 
graduelle de techniques ou de produits existants. Elle ne bouleverse pas les conditions d'usage, mais y apporte une sensible amélioration. Elle ne change pas fondamentalement la dynamique d'une entreprise.

Innovation de valorisation : elle engendre un changement important par rapport à la simple optimisation : augmentation importante en termes de créativité et d'originalité. Il s'agit de mettre en œuvre de nouvelles combinaisons possibles de compétences déjà maitrisées, qui sont propres à l'entreprise et liées aux données auxquelles elle a accès. Ces nouvelles combinaisons sont donc spécifiques de chaque entreprise en fonction de son histoire et de son identité propre.

Innovation de rupture, absolue ou radicale : il s'agit là du résultat d'un processus de recherche (interne ou externe) ou d'un acte de " génie créatif », suivi d'une mise en œuvre et d'un passage à l'acte. L'acte d'innovation est absolu tant du point de vue interne (entreprise ne l'ayant jamais fait), qu'externe (aucune autre entreprise ne l'ayant fait avant). Dans cette démarche, il y a une rupture avec l'existant, une réelle création avec un niveau d'enjeu important, puisque l'adoption de l'innovation entraîne une modification radicale des conditions d'utilisation d'un produit et bouleverse les habitudes.

b) Les formes d'innovation, selon l'objet qu'elle modifie

Les innovations technologiques sont des innovations à caractère le plus souvent « segmenté ». Elles ne ne remettent pas fondamentalement en cause le fonctionnement de l'entreprise. Elles correspondent à l'introduction d'un nouvel objet (technologique/technique/produit) ou d'un objet existant, mais incluant une nouveauté. La plupart d'entre elles a été conçue à partir d'inventions préalables (Rammel et Van den Bergh 2003, Boiffin et al 2004, OCDE 2010).

Les innovations organisationnelles permettent l'amélioration, la mise au point ou l'adoption de méthodes d'organisation de la production, du lieu de travail ou de distribution de l'entreprise. Elles contribuent à modifier les processus de production et l'organisation du travail. Elles ne correspondent pas à l'introduction d'un objet nouveau, comme dans l'innovation technologique, mais à de nouvelles pratiques, à de nouveaux agencements entre les acteurs de l'entreprise (Deltour 2000, OCDE 2010).

Les innovations systémiques engendrent une restructuration importante ou une reconfiguration plus ou moins profonde du système de production. Elles aboutissent à une nouvelle cohérence fonctionnelle et stratégique. Elles touchent pratiquement toutes les fonctions de l'entreprise (Boiffin et al 2004, Meynard et al 2006, Giget 2007).

Les innovations de commercialisation : ce sont des innovations qui consistent à développer une nouvelle méthode de commercialisation impliquant des changements significatifs de la conception ou du conditionnement, du placement, de la promotion ou de la tarification d'un produit (OCDE 2010). Ces innovations sont très importantes, car elles se situent au carrefour de ce que produit l'entreprise (gamme) et de la demande du marché. Elles sont au cœur de la flexibilité de l'entreprise, prise sous l'angle de sa sensibilité aux aléas des prix.

Les innovations institutionnelles : elles concernent la mise en place de nouveaux arrangements ou mécanismes (une nouvelle architecture institutionnelle) au niveau de la distribution du pouvoir et de contrôle des ressources de la politique publique (Lévesque 2005). Elles sont fondamentalement collectives, et les entreprises ont plus ou moins « la main » pour en déterminer les orientations (cf. la cogestion pendant des années en France entre le pouvoir politique et le syndicalisme agricole).

\section{3 / Illustrations des change- ments induits en élevage selon le type d'innovation}

Dans la littérature, les questions posées et les réponses données diffèrent selon le type d'innovation étudié. Par exemple, les facteurs facilitant la mise en œuvre d'une innovation systémique radicale ne sont pas forcément les mêmes que pour une innovation technologique incrémentale. Il est donc essentiel d'identifier à la fois le ou les types d'innovation considérés, leur combinaison et les facteurs mis en jeu pour mieux comprendre le processus d'innovation (figure 4).

\section{1 / Les innovations technologi- ques}

En élevage, de nombreuses innovations technologiques relèvent d'une logique incrémentale, comme dans le cas de nouvelles générations de matériels vivants (nouvelles variétés végétales, nouveaux génotypes animaux) ou de machines et d'équipements d'élevage. La logique est ici doublement incrémentale : ces nouveautés sont développées par les entreprises de l'agrofourniture, selon une activité de type exploitation, où l'innovation «consiste à faire mieux ce qu'on sait déjà faire » (Mothe et Brion 2008), et utilisées par les éleveurs, sans qu'elles ne remet- tent en question le fonctionnement des ateliers d'élevage ou l'organisation globale du système de production.

Les innovations technologiques peuvent également être des innovations de rupture : le développement de l'Insémination Artificielle (IA) dans le secteur laitier a ainsi contribué à l'essor des schémas d'amélioration génétique, avec la mise place de nouveaux opérateurs (centre d'insémination, organisme de sélection), organisés et réglementés dans le cadre de la loi sur l'élevage de 1966. L'usage de l'IA en ferme a modifié profondément le fonctionnement des élevages bovins laitiers dans les années 19501960, mais aussi les réseaux de relations entre les éleveurs organisés autour des échanges d'animaux pour la reproduction. Entre les années 1980 et 2000, de nombreuses innovations ont été mises en œuvre chez les opérateurs des schémas de sélection laitiers. Ces innovations ont porté sur les technologies de la reproduction, comme l'ovulation multiple et le transfert d'embryons (Colleau 1993) et sur l'évaluation génétique des animaux reproducteurs, comme l'utilisation des marqueurs moléculaires pour détecter des «Quantitative Trait Loci » (QTL) dans les 3 grandes races laitières françaises (Boichard et al 2000, Fritz et al 2003). Ces innovations ont permis d'améliorer la performance des schémas de sélection; elles sont plutôt incrémentales, n'ayant pas bouleversé le fonctionnement de ces schémas. Elles n'ont pas entraîné d'innovations en ferme chez les éleveurs, toujours utilisateurs de semences de taureaux aux valeurs génétiques très précisément connues, grâce au testage sur descendance. En revanche, à partir de la fin des années 2000, la sélection génomique (Fritz et al 2010) est une innovation de rupture, dans la mesure où elle induit la suppression du testage sur descendance, ce qui change profondément le fonctionnement des entreprises de sélection, avec la commercialisation de semences de jeunes taureaux, dont l'évaluation génétique est moins précise qu'auparavant. Pour les éleveurs, cette innovation entraîne une modification dans les pratiques de choix des taureaux (fin du « star system », Colleau et al 2009), mais ne bouleverse ni la logique de fonctionnement des élevages laitiers, ni la logique de l'amélioration génétique reposant essentiellement sur la voie mâle. Le génotypage des femelles, service proposé aux éleveurs, ou l'évaluation sur de nouveaux critères pourraient entraîner des usages en ferme relevant d'innovations de rupture, mais il est encore trop tôt pour le dire.

Les innovations technologiques dans les industries de l'agrofourniture peuvent également entraîner des innovations de 
Figure 4. Représentation du classement des innovations selon l'objet qu'elles modifient et selon le degré de changement qu'elles induisent. Les exemples positionnés sur la figure sont décrits dans le texte.

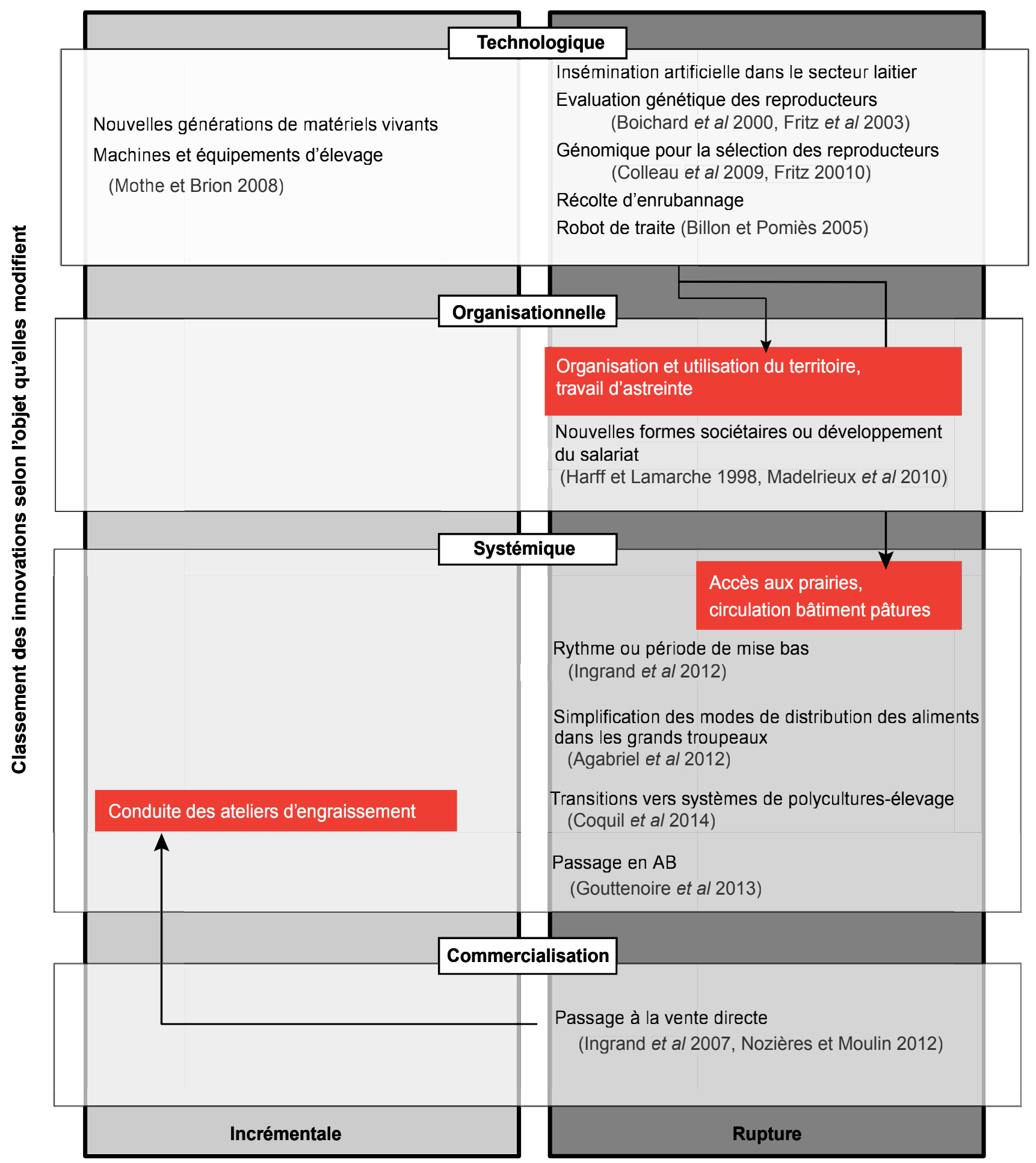

Classement des innovations selon le degré de changement induit

rupture dans les systèmes d'élevage. C'est le cas par exemple des innovations sur la récolte des fourrages (presses à balle ronde, enrubannage) ou sur la traite (robots). Ces innovations technologiques entraînent des modifications dans les processus de production. Par exemple, l'enrubannage permet de réaliser des premières coupes plus précoces par rapport à la fauche pour le foin, sur des superficies plus réduites que celle nécessaires pour un chantier d'ensilage. L'utilisation des différentes parcelles de l'exploitation est ainsi modifiée et est associée à un accroissement de la quantité et la qualité des fourrages produits. L'enrubannage donne également de la souplesse pour le stockage des fourrages conservés. L'introduction de ces innovations technologiques a également des conséquences sur l'organisation du travail. 
Par exemple, le robot de traite permet de supprimer l'astreinte de la présence de l'éleveur pour la traite, et entraîne en conséquence des modifications sur les autres travaux d'élevage et la vie de famille (Billon et Pomiès 2006). De même, les modes d'organisation et d'utilisation du territoire de l'exploitation peuvent également être modifiés. Ainsi, pour maintenir un rythme de deux traites quotidiennes en période de pâturage, le recours à un robot de traite nécessite l'aménagement en conséquence des accès aux prairies et de la circulation entre le bâtiment et les parcelles pâturées (Billon et Pomiès 2006). Ainsi, l'adoption d'une innovation technologique en ferme entraîne des innovations de type organisationnel et finalement systémique, correspondant à une innovation de rupture en ferme.

\section{2 / Les innovations organisation- nelles et commerciales}

Les processus d'intensification et de spécialisation des productions animales ont souvent été accompagnés, favorisés, voire déclenchés par des innovations technologiques. Cependant, les agriculteurs mettent en œuvre également de nombreuses innovations qui ne font pas intervenir de nouveaux objets techniques. Les modifications de rythme ou de période de mise bas en élevage allaitant (Ingrand et al 2012), la simplification des modes de distribution de l'alimentation dans les grands troupeaux (Agabriel et al 2012) en sont des exemples. La transition, en zone de plaine, vers des systèmes de polyculture-élevage plus autonomes et économes en intrants (Coquil et al 2014) en est également une bonne illustration, au même titre que la conversion à l'agriculture biologique, déjà citée. L'innovation est ici de type systémique, remettant en question l'ensemble du système de production (nécessité d'une re-conception globale). A l'échelle des groupes qui s'inscrivent dans un tel processus, il s'agit d'une innovation radicale, touchant la conduite des activités productives, mais également les normes professionnelles. Par exemple, l'usage du pâturage tournant dans des exploitations en transition vers des systèmes autonomes "provoque une modification profonde des façons de travailler et de mobiliser le conseil » (Coquil et al 2014).

Les nouvelles formes sociétaires ou le développement du salariat dans les exploitations d'élevage (Harff et Lamarche 1998, Madelrieux et al 2010) sont également des innovations de nature orga- nisationnelle. Le passage à la vente directe dans les systèmes allaitant, que ce soit en élevage bovin (Ingrand et al 2007) ou en élevage ovin (Nozières et Moulin 2012), est un exemple d'innovation de commercialisation. Si l'organisation du collectif de travail ou la commercialisation des produits de l'élevage peuvent être les cibles premières de l'innovation, la conduite des activités s'en trouve souvent modifiée, entraînant une innovation de forme systémique. Cette dernière peut rester alors incrémentale, comme le montre l'étude d'Ingrand et al (2007), rapportant comment la mise en place de la vente directe modifie la production, via les volumes d'animaux finis et les conduites en ateliers d'engraissement, mais sans bouleversement notable.

Il est important de noter que ce processus d'innovation organisationnelle touchant la conception des systèmes implique une forme spécifique d'organisation du dispositif de recherchedéveloppement (par rapport au dispositif associé à l'innovation technologique). Le prototypage de nouveaux systèmes et l'expérimentation-système (Coquil et Ingrand 2008) sont des outils privilégiés de la recherche agronomique pour participer à ce processus. Ces outils se sont développés de manière significative des dernières années en France, comme la plateforme Inra de recherches en production ovine allaitante en Agriculture Biologique (Prache et al 2011), les expérimentations système mises en place sur le long terme et impliquant des collectifs de recherche pluridisciplinaires ou encore le pilote mis en place au Lycée Agricole de Guadeloupe pour développer des systèmes de polyculture-élevage visant à augmenter le niveau de production par unité de surface cultivée (Fanchone et al 2013). Ainsi, on peut dire que pour générer l'innovation systémique chez les agriculteurs, le dispositif de recherche-développement doit lui-même opérer un processus d'innovation interne de même nature.

\section{Conclusion}

Les innovations correspondent à l'introduction dans un système existant, d'une nouveauté, technologique ou non, dans le cadre d'un processus qui peut prendre du temps. Le caractère nouveau est vu au regard des systèmes pratiqués localement, voire au regard de celui qui innove, relativement à ce qu'il faisait avant. Enfin, le processus d'innovation n'est pas forcément synchronisé dans le temps et dans l'espace.
L'objet, voire la finalité de l'innovation, peut être amené à se transformer au cours du processus. En outre, par des effets en cascade, l'introduction d'une nouveauté dans un système entraîne souvent des modifications sur l'ensemble du système, pour atteindre une nouvelle cohérence. Ces effets induits, ainsi que l'appréciation des gains potentiels par celui qui innove, peuvent expliquer l'acceptabilité plus ou moins grande des innovations que les structures de recherche-développement ont conçues et proposent. Ainsi, les innovations en élevage sont évaluées par les éleveurs eux mêmes, mais aussi par les membres de la société civile, qui peuvent y être réfractaires.

Comment la recherche peut-elle alors être plus efficace pour contribuer au processus d'innovation en élevage, notamment lorsque des démarches participatives sont mises en œuvre ? Nous pensons qu'elle peut renforcer la capacité à innover dans le cadre de réseaux d'éleveurs, en créant des dispositifs de repérage de nouvelles pratiques, le plus souvent parcellaires ou fragmentaires, mais qui peuvent être mobilisées pour concevoir des systèmes en rupture, comme cela a été le cas par exemple pour les systèmes fourragers innovants (brainstorming d'un groupe de chercheurs appuyé par six départements de l'Inra, ayant abouti à la mise en place d'une expérimentation système à Lusignan). La recherche a les moyens également d'aider à l'évaluation multicritère de pratiques et de systèmes, en mobilisant différents outils, tels que la modélisation, les essais en station (cf. les expérimentations système citées précédemment) et les réseaux de fermes privées...), en lien avec les structures de recherche/développement (dans le cadre de dispositifs partenariaux tels que les Réseaux Mixtes Thématiques et des Unités Mixtes Technologiques).

Enfin, il s'agit de prendre en compte explicitement les freins à l'innovation en élevage (ou en contrepoint, les conditions de leur acceptabilité), à la fois dans le monde de l'élevage et dans la société civile. Des démarches de ce type sont en cours à l'Inra, avec un groupe de travail pluridisciplinaire récemment constitué autour de la problématique de la perception par les porteurs d'enjeux de la société civile, des pratiques d'élevage et des biotechnologies animales. Nous pensons, qu'en retour, des idées nouvelles peuvent émerger pour imaginer, tester, mettre en place des innovations, dans le cadre d'un processus participatif. 


\section{Références}

Agabriel J., Farrié J.P., Pottier E., Note P., 2012. Simplifications de l'affouragement pour les troupeaux allaitants: évaluation des pratiques et de leurs conséquences zootechniques. Renc. Rech. Rum., 19, 385-392.

Akrich M., Callon M., Latour B., 1988. A quoi tient le succès des innovations ? 1: L'art de l'intéressement, Gérer et comprendre, Annales des Mines, 11, 4-17.

Alter N., 2010. L'innovation ordinaire. PUF Editions, Paris, France, $324 \mathrm{p}, 3^{\text {ème }}$ édition.

Ameur F., Hamamouche M.F., Kuper M. Benouniche M., 2013. La domestication d'une innovation technique : la diffusion de l'irrigation au goutte-à-goutte dans deux douars au Maroc. Cah. Agric., 22, 311-318.

Bal P., Castellanet C., Pillot D., 2003. Faciliter l'émergence et la diffusion des innovations. in CIRAD-GRET, Le Mémento de l'Agronome. QUAE Editions, Versailles, France.

Barruentes-Fuentes J.C., Berg E., 2013. Impact assessment of agricultural innovations: a review. Agronomia Colombiana, 31, 120-130.

Beguin P., Griset P., Licoppe C., Mallein P., 2008. Innovations et conceptions, usages et savoirs-faires, produits et objets. Science \& Devenir de l'homme. Les Cahiers du M.U.R.S, 57-58, 218-240.

Bentz B., Gretz P.E., 2002. Appuyer les innovations paysannes. Saint-Etienne : Edition du GRET.

Billon P., Pomiès D., 2006. Le point sur la robotisation de la traite 15 ans après l'apparition des premiers systèmes dans les fermes. Renc. Rech. Rum., 13, 143-150.

Binh V.T., 2010. Le projet DURAS : des partenariats innovants pour le développement durable. Extrait du dossier thématique d'Agropolis International- spécial partenariat, p48.

Bocquier F., Debus N., Lurette A., Maton C., Viudes G., Moulin C.H., Jouven M., 2014. Elevage de précision en systèmes d'élevage peu intensifiés. In: Numéro spécial, Quelles innovations pour quels systèmes d'élevage ? INRA Prod. Anim., 27, 101-112.

Boichard D., Grohs C., Bourgeaois F., Cerqueiras F., Faugeras R., Neau A., Milan D., Rupp R., Amigues Y., Boscher M.Y., Leveziel H., 2000. La recherche de QTL à l'aide de marqueurs : résultats chez les bovins laitiers. In : Numéro hors-série, Génétique moléculaire : principes et application aux populations animales. INRA Prod. Anim., 13, 217-222.

Boiffin J., Hubert B., Durand N., 2004. Agriculture et Développement Durable. Enjeux et questions de recherche. INRA Editions, Paris, France, 91p.

Bourbouze A., 2006. Systèmes d'élevage et production animale dans les steppes du nord de l'Afrique: une relecture de la société pastorale du Maghreb. Sécheresse, 17, 31-39.

Bouyer F., Belem A., Seyni H., Adakal H., Lancelot R., Stachursky F., Bouyer J., 2011a. Adoption d'une nouvelle méthode de lutte sanitaire en milieu paysan : le pédiluve acaricide/ insecticide au Burkina Faso. Rev. Elev. Med. Vet. Pays Trop., 64, 10p.

Bouyer F., Hamadou S., Adakal H., Lancelot R., Stachurski F., Belem A.M.G. Bouyer J., 2011b. Restricted Application of Insecticides: A Promising Tsetse Control Technique, but
What Do the Farmers Think of It? PLoS Negl Trop Dis 5(8): e1276. doi:10.1371/journal. pntd. 0001276.

Callon M., Latour B., 1990. La science telle qu'elle se fait. Editions La Découverte, Paris, France, $868 \mathrm{p}$.

Chia E., 2004. Principes, méthodes de la recherche en partenariat: une proposition pour la traction animale. Rev. Elev. Med. Vet. Pays Trop., 57, 233-240.

Colleau J.J., 1993. Les biotechnologies de 1'embryon bovin : application à la selection, réalités et enjeux économiques. Cah. Agric., 2, 93-102.

Colleau J.J., Frtiz S., Guillaume F., Baur A., Dupassieux D., Boscher M.Y.N., Journaux L., Eggen A., Boichard D., 2009. Simulation des potentialités de la sélection génomique chez les bovins laitiers. Renc. Rech. Rum., 16, 419.

Coquil X., Ingrand S., 2008. Prototyping sustainable dairy systems by evaluating their flexibility and plasticity in a system experimentation. $8^{\text {th }}$ Eur. IFSA Symp., 6 - 10 July 2008 , Clermont-Ferrand, France, 507-509.

Coquil X., Béguin P., Dedieu B., 2014 Systèmes de polyculture élevage laitiers évoluant vers l'AB Renforcement des interfaces cultures/élevage. Econ. Rurale, 339-340.

Cros F., 1997. L'innovation en éducation et en formation. Revue française de pédagogie, 118 , 127-156.

Dagenais C., Desbiens F., Joubert P., 2005 L'expérience du FASS au Québec : une occasion d'apprentissage sur le processus d'introduction de l'innovation et de gestion du changement. La Revue de L'innovation: La Revue de l'innovation dans le secteur public, 10 , article $6,17 \mathrm{p}$.

Dalglish C, Newton C, 2002. The relationship between firm survival and innovation: An introduction to the literature. Innovation: Management, Policy \& Practice, 4, 209-214

Darnhofer I., Bellon S., Dedieu B., Milestad, R., 2010. Adaptiveness to enhance the sustainability of farming systems. A review. Agron. Sustainable Develop., 30, 546-555.

Darré J.P., 1996. L'invention des pratiques dans l'agriculture. Vulgarisation et production locale de connaissance. Khartala, Paris, France, 194p.

Davis S.R., Farr V.C., Stelwagen K., 1999. Regulation of yield lossband milk composition during once-a-day milking: a review. Livest. Prod. Sci., 59, 77-94.

Delerce S., Moulin C.H., Tchakérian E., 2011. "Solutions collectives d'adaptation aux aléas climatiques pour les élevages de la zone périméditerranéenne" FOCUS PSDR3 (http:// www6.inra.fr/psdr-midi-pyrenees/FilActus/Dernieres-publications-PSDR/FocusPSDR3-Climfourel-Solutions-collectives-dadaptation-aux-aleas-climatiques, consulté le 12/12/2013

Deltour F., 2000. L'innovation dans l'organisation : dépasser les ambiguïtés du concept. Les Cahiers de la Recherche, Centre Lillois d'Analyse et de Recherche sur l'Evolution des Entreprises, UPRES-A CNRS 8020, 1-18.

Diallo A., 1994. La transformation des produits agricoles locaux au Burkina Faso : des innovations en échec. Centre ORSTOM de Ouagadougou Programme de recherche: «politiques et développement agricole et agro-industriels au
Burkina Faso ». Responsable : Y.-A. FAURE, Document $n^{\circ} 2$, Février 1994.

Dockès A.C., Morhain B., Tchakérian E., 2004. Accompagner les éleveurs dans les processus de changement technique, les méthodes et les outils de l'Institut de l'Elevage. Institut de l'élevage, article ref $n^{\circ} 240459030$.

Dogliotti S., García M.C., Peluffo S., Dieste J.P., Pedemonte A.J., Bacigalupe F.C., Scarlato M., Alliaume F., Alvarez J., Chiappe M., Rossing W.H.A., 2014. Co-innovation of family farm systems: A systems approach to sustainable agriculture. Agricult. Sys., 126, 76-86.

Duru M., Cruz P., Jouany C., Théeau J.P., 2011. Combiner des recherches en agroécologie et des dispositifs participatifs pour construire des outils d'évaluation des prairies permanents. Cah. Agric., 20, 223-234.

Elzen B., Geels F.W., Green K., 2004. System innovation and the transition to sustainability: theory, evidence and policy. Edward Elgar Publishing, Cheltenham, 315p.

Fanchone A., Stark, F., Kelemen J.L., Alexandre G., Diman J.L., Gourdine J.L., Ozier-Lafontaine H., Sierra J., Tournebize R., Archimede H., 2013. Conception d'un système pilote de type polyculture élevage et test d'innovations au lycée agricole (EPLEFPA) de Guadeloupe. Séminaire ACTA-INRA, les systèmes de polyculture en élevage dans les territoires, Toulouse, France.

Flichy P., 1995. L'innovation technique : récents développements en sciences sociales. Vers une nouvelle théorie de l'innovation. Editions La Découverte, Paris, France, 207p.

Frank B., 1995a. Contrainsts limiting innovation adoption in the North Queensland beef indudstry. I : A sosio-economic means of maintaining a balanced lifestyle. Agricult. Sys., 47, 291-321

Frank B., 1995b. Contrainsts limiting innovation adoption in the North Queensland beef industry. II : Non-adoption is an intelligent response to environnemental circumstances. Agricult. Sys. 47, 323-346.

Frank B., 1997. Adoption of innovations in the north Queensland beef industry. 3. Implications for extension management. Agricult. Sys., 55 , $347-358$

Fritz S. Colleau J.J., Druet T., Boscher M.Y., Rossignol M.N., Malafosse A., Boichard D., 2003. Mise en place d'une Sélection Assistée par Marqueurs dans les trois principales races bovines laitières françaises. Renc. Rech. Rum., 10, 53-56.

Fritz S., Guillaume F., Croiseau P., Baur A., Hoze C., Dassonneville R., Boscher M.Y., Journaux L., Boichard D., Ducrocq V., 2010. Mise en place de la Sélection Génomique dans les trois principales races françaises de bovins laitiers. Renc. Rech. Rum., 17, 455-458.

Geels F., 2011. The multi-level perspective on sustainability transitions: Responses to seven criticisms. Environ. Innov. Soc.Transit., 1, 2440 .

Giget M., 2007. L'innovation dans l'entreprise. Techniques de l'ingénieur.

Gonard T., Louazel M., 1997. « Comprendre les processus d'innovation technique à l'aide du concept de réseau : un programme de recherche ", Cahier de Recherche du CREA, $\mathrm{n}^{\circ} 62$, juin 1997. 
Goulet F., Pervanchon F., Cerf M., 2008. Les agriculteurs innovent par eux mêmes pour leur système de culture. Reau Raymond D.T. (Ed), Systèmes de culture innovants et durables: quelles méthodes pour les mettre au point et les évaluer? Educagri, Dijon, France, 53-69.

Gouttenoire L., Cournut S., Ingrand S., 2013. Farm-scale models built with farmers converting and converted to organic farming. Agron. Sustain. Dev., 33, 609-619.

Harff Y., Lamarche H., 1998. Le travail en agriculture : nouvelles demandes, nouveaux enjeux. Econ. Rurale, 244, 3-11.

Ingrand S., Limon M., Veysset P., 2007. La vente directe en élevage bovin viande : résultats économiques et conséquences sur la conduite technique. Renc. Rech. Rum., 14, 453.

Ingrand S., Devun J., PAilleux J.Y., Chauvet A., Dujour E., 2012. Les innovations en élevages bovins et ovins allaitants : analyse de résultats d'entretiens auprès d'éleveurs et de réponses d'experts à un questionnaire. Renc. Rech. Rum., 19, 393-396.

Jullian P., 1989. Innovations et diversité des exploitations agricoles. Econ. Rurale ,192-193, 104-109.

Landais E., Deffontaines J.P., 1988. Les pratiques des agriculteurs. Point de vue sur un courant nouveau de la recherche agronomique. Etudes Rurales, 125-158.

Landais E., Lhoste P., 1990. L'association agriculture-élevage en Afrique intertropicale : un mythe techniciste confronté aux réalités du terrain. Cah. Sci. Hum. 26, 277-235

Lasseur J., 2005. Sheep farming systems and nature management of rangeland in French Mediterranean mountain areas. Livest. Prod. Sci., 96, 87-95.

Le Bail M., Meynard J.M., 2003. Yield and protein concentration of spring malting barley: the effects of cropping systems in the Paris Basin (France). Agronomie, 23, 13-27.

Leeuwis C., Van Den Ban A., 2004. Communication for Rural Innovation: Rethinking Agricultural Extension. Third Edition. Oxford : Blackwell Publishing, 428p.

Lefort J., 1988. Innovation technique et expérimentation en milieu paysan. Cah. Rech. Dev., $17,1-10$.

Lemarié S., Joly P.B., 2000. « Cinquante d'innovations en agriculture : quelques enseignements des sciences socio-économiques ». Econ. Rurale, 255-256, 86-97.

Lévesque B., 2005. Innovations et transformations sociales dans le développement économique et le développement social : approches théoriques et politiques publiques. Cahier du CRISES, Collection Etudes Théoriques, p72.

Lynne G. D., 1995. Modifying the neo-classical approach to technology adoption with behavioral science models. J. Agricult. Appl. Econ., 27, 67-80.
Lynne G.D., Shonswiler J., S., Rola L.R., 1988. Attitudes and farmer conservation behavior. American J. Agricult. Appl. Econ., 70,12-19.

Madelrieux S., Dupré L., Hostiou N., Barbosa T., Burlamaqui Bendahan A., Tourrand J.F., 2010. Liens entre salariat et activité agricole: itinéraires professionnels de salariés d'élevage. Cah. Agric., 19, 354-358.

Marguin L., Balvay B., 2009. Interest of Electronic Identification for ruminants. ICAR Meeting, Porec, Croatie, 2009 May $13^{\text {th }}$.

Matanmi B.M., 1994. Some criteria in acceptance of agricultural innovations by small scale farmers in Nigeria. NOMA Magazine, 11, 28-30.

Meynard, J.M., Aggeri F., Coulon J.B., Habib R., Tillon J.P., 2006. Conception de systèmes agricoles innovants. Rapport à la Direction de l'INRA, $55 \mathrm{p}$

Meynard J.M., DourmadJ.Y., 2014. L'innovation en élevage : de nouvelles démarches pour de nouveaux enjeux. In : Numéro spécial, Quelles innovations pour quels systèmes d'élevage ? Ingrand S., Baumont R. (Eds). INRA Prod. Anim., 27, 77-88.

Milleville P., 1991. Systèmes d'élevage. In : Claude J., Grouzis M., Milleville P. (Eds). Un espace sahélien: la mare d'Oursi. Burkina Faso. ORSTOM Editions, Paris, France, 155178.

Montaigne E., Cadot J., 2006. L'innovation dans la filière vitivinicole en LanguedocRoussillon. In : Dynamiques des entreprises agroalimentaires (EAA) du LanguedocRoussillon : évolutions 1998-2003. Série Cahier de Recherche (3). Montpellier, FRA : INRAUMR MOISA, 89-101.

Mohr L.B., 1982. Explaining organizational behavior: the limits and possibilities of the-ory and research. San Francisco: Jossey-Bass Inc.

Mothe C., Brion S., 2008. Innovation : exploiter ou explorer? Rev. Française Gestion, 187, 101-108.

Moulin C.H., Forel E., Lelièvre F., 2009. Autonomie et robustesse des systèmes d'élevage en zone périméditerranéenne face aux évolutions de la variabilité climatique. Renc. Rech. Rum., 16, 377.

Nonaka I., Takeuchi H., 1997. Théorie de la création de connaissances organisationnelles, In: La connaissance créatrice. La dynamique de l'entreprise apprenante, chapitre 3, De Boeck Université, Louvain-la-Neuve, Belgique.

Nozières M.O., Moulin C.H., 2012. Quelles formes de vente directe des agneaux en Languedoc Roussillon ? Renc. Rech. Rum., 19, 306.

OCDE, 2010. Mobiliser l'innovation pour affermir la croissance et relever les défis planétaires et sociaux : Principales constatations. www.oecd.org/innovation/strategy, Consulté le $0311,2011$.

Pichot J.P., Faure G., 2008. Systèmes d'innovations et dispositifs d'appui pour les agricul- tures africaines subsahariennes. In: Devèze J.C. (Ed). Défis agricoles africains. Karthala, Paris, France, 265-284.

Pottier E., Devun J., Farriè J.P., Ingrand S. Pailleux J.Y., D'Hour P. Mialon M.M., Brule A., Solas L., Renon J., Note P., 2014. Systèmes allaitants et innovations en élevage. Innov. Agronom. 34, 207-226.

Prache S., Benoit M., Tournadre H., Cabaret J., Laignel G., Ballet J., Thomas Y., Hoste H. Pellicer M., Andueza D., Hostiou N., Giraud J.M., Sepchat B., 2011. Plateforme INRA de recherches en production ovine allaitante $\mathrm{AB}$ : de l'étude de verrous techniques à la conception de systèmes d'élevage innovants. Renc. Rech. Rum., 18, 61-64.

Rammel C., Van den Bergh J., 2003 Evolutionnary policies for sustainable development : adaptive flexibility and risk minimising Ecol. Econom., 47, 121-133.

Rogers E., 1962. Diffusion of innovations. New York: The Free Press, 453p.

Sabourin E., Da Silveira L.M., Sidersky P., 2004. Production d'innovation en partenariat et agriculteurs-expérimentateurs au Nordeste du Brésil. Cah. Agric., 13, 203-210.

Schroeder R.G., Van de Ven A., Scudder G. D., Polley D., 1989. The development of innovation ideas. In: Van de Ven A., Angle H.L., Poole M. (Eds). Research on the management of innovation: The Minnesota studies. New York: Harper \& Row.

Schumpeter J., 1935. Théorie de l'évolution économique. Recherches sur le profit, le crédit, l'intérêt et le cycle de la conjuncture. (nouvelle édition 1983). Dalloz, Paris, France, 589p.

Sørensen K.H., 2006. Domestication : the enactment of technology. In: Berker T. Hartmann M., Punie Y., Ward K.J. (Eds). Domestication of Media and Technology. London, UK. Open University Press.

Sulpice P., Pichard G., Dufour A., 2006. Les éleveurs construisent leur savoir en groupe. Travaux \& Innovations, 127, 16-20

Van de Ven, A.H., Poole M.S., 1995 'Explaining development and change in organizations', Acad. Management Rev., 20, 510-540.

West M., Altink WMM., 1996. Innovation at work: Individual, group, organizational and socio-historical perspectives. Europ. J. Work Organizat. Psychol., 3-11.

Willinger M., Zuscovitch E., 1993. Efficience, irréversibilités et constitution des technologies. Rev. Econ. Industr., 65, 7-22.

Willock J., Deary I.J., Edwards-Jones J.B., Gibson G.J., McGregor M.J., Sutherland A., Dent, J.B. Morgan W., Grieve R., 1999. The role of attitudes and objectives in farmer decision making: Business and environmentallyoriented behaviour in Scotland. J. Agricult. Econ., 50, 286-303. 


\title{
Résumé
}

Le terme « innovation » est largement utilisé, notamment dans le domaine de la recherche. L'innovation se démarque de l'invention, processus en amont, et se caractérise par différentes modalités de mise en place de quelque chose de nouveau dans un système existant. Les sources de l'innovation peuvent être exogènes à la structure qui innove, ou bien endogènes dans le cas où l'innovation est initiée par celui qui pilote la structure (ici les exploitations agricoles). En agriculture, et en élevage en particulier, les innovations organisationnelles (faire autrement avec les mêmes choses) sont aussi importantes que les innovations technologiques (utiliser un objet nouveau ou un ancien objet modifié), notamment quand les animaux (en tant qu'êtres vivants) sont directement en jeu. Le type d'innovation considéré, ainsi que le degré et la nature du changement qui lui sont associés, sont essentiels dans la compréhension des processus d'innovation. De même, les facteurs favorables ou, au contraire, défavorables à ce processus, doivent être identifiés afin d'y contribuer efficacement en tant que chercheurs. Des exemples pris dans le domaine de l'élevage, en France ou ailleurs dans le monde, illustrent les différentes notions liées à l'innovation, tout au long du texte.

\begin{abstract}
What is on-farm innovation process? Some examples in livestock farming systems.

The term " innovation " is widely used, particularly in the field of research. Innovation differs from invention, and is characterized by different modalities of implementing something new in an existing system. The sources of innovation may be exogenous to the structure, or endogenous where innovation is initiated by the person who controls the structure (here the farms). In agriculture and animal husbandry in particular, organizational innovations (ie do differently by using the same components) are as important as technological innovations (use a new object), especially when the animals are directly concerned. The type of innovation considered, as well as the degree and nature of change associated with it, are essential to understand the innovation process. Similarly, favorable or unfavorable factors must be identified by researchers in order to contribute effectively to this innovative process. Examples in livestock farming systems, in France or elsewhere in the world, illustrate the various concepts related to innovation throughout the text.
\end{abstract}

INGRAND S., LURETTE A., GOUTTENOIRE L., DEVUN J., MOULIN C.-H., 2014. Le processus d'innovation en ferme. Illustrations en élevage. In : Numéro spécial, Quelles innovations pour quels systèmes d'élevage ? Ingrand S., Baumont R. (Eds). INRA Prod. Anim., 27, 147-160. 